\title{
Verifying the deicing capacity of superhydrophobic anti-icing surfaces based on wind and thermal fields
}

\author{
Chunling Zhu ${ }^{\mathrm{a}}$, Senyun Liu ${ }^{\mathrm{a}}$, Yizhou Shen ${ }^{\mathrm{a}, \mathrm{b}, *}$, Jie Tao $^{\mathrm{b}}$, Guanyu Wang $^{\mathrm{b}}$, Lei Pan ${ }^{\mathrm{b}}$ \\ a College of Aerospace Engineering, Nanjing University of Aeronautics and \\ Astronautics, 29 Yudao Street, Nanjing 210016, P. R. China \\ ${ }^{b}$ College of Material Science and Technology, Nanjing University of Aeronautics and \\ Astronautics, 29 Yudao Street, Nanjing 210016, P. R. China
}

*Corresponding author: (Y. Shen) E-mail: shenyizhou@ nuaa.edu.cn 
Abstract: Based on the high icing-delay performance and low ice adhesion strength, the anti-icing potential of superhydrophobic surfaces has been well investigated over the past years. The aim of this work was to verify the deicing capacity of superhydrophobic surfaces under the conditions of wind and thermal fields, expecting to promote its engineering applications. We took various wetting surfaces ranging from hydrophilic to superhydrophobic as research objects, and discussed the deicing properties of these sample surfaces based on the wind and thermal fields. The results indicated that under the condition of wind field, the micro-nanoscale hierarchical structured superhydrophobic surface exhibited a remarkable deicing property (no matter what the freezing temperature was), due to the time required for blowing away ice on the surface being least. However, the specific micro-nanoscale hierarchical structures on the superhydrophobic surface also induced a poorly melting ice property owing to the lower heat transfer efficiency caused by trapped air pockets. Furthermore, the superhydrophobic surface displayed a robust durability under the condition of 30 icing/deicing cycles. Therefore, we believe that the wind field deicing is an ideal choice as the assistant deicing approach considered in the practical applications of the anti-icing superhydrophobic surfaces.

Keywords: superhydrophobic; deicing; anti-icing; wind field; thermal field 


\section{Introduction}

Undesired ice accretion has brought many inconveniences to our daily lives, even leading to severe economic impacts and large losses of lives, such as aircraft icing endangering the safety of flights, road icing affecting the public transport, ice downing power lines, and ice accumulation in refrigerators and heat exchangers resulting in the decrease of heat transfer efficiency [1-6]. In order to retard or prevent the ice build-up, many efforts have been made to understand the underlying physicochemical mechanism of icing, and develop many anti-icing and deicing strategies over the past decades [7-9]. Most strategies are divided into the active and passive anti-icing/deicing techniques. Currently, the active deicing approaches, such as scattering ice-melting agent, mechanical vibration, and electro-thermally melting ice, have been widely used in the aircraft industry, power transmission and daily lives based on the purposes of melting and breaking ice [10-13]. However, these popular methods generally rely on continuous heating or vibration of the icing locations, and the corresponding equipments are also hard to design, which leads to these methods being gradually eliminated in the future [14]. Fortunately, accompanying with the rise of bionic technique, the anti-icing superhydrophobic (contact angle greater than $150^{\circ}$ and contact angle hysteresis less than $10^{\circ}$ ) surface as the passive anti-icing technique has been proposed and widely investigated for the ideal applications without any energy consuming, which also becomes one of the most significant research topics recently [15-17].

Generally, icing on a solid surface begins with the wetting of supercooled water 
droplets in the environment of low temperature. This wetting process is considered to play a significant role to determine the feasibility of icing, where the solid/water interface gradually replaces the solid/air interface $[18,19]$. Thus, the non-wetting characteristic is a crucial factor to prevent icing on a solid surface. Bio-inspired superhydrophobic surfaces have recently aroused researchers' intense attentions for the applications in anti-icing field, precisely because of their remarkable non-wettability [20-22]. The non-wettability mainly relies on the cooperation of surface free energy and surface texture. One of the most effective ways of controlling the surface free energy is to covalently graft polymer hydrophobic groups or chains on the solid surface [23]. Currently, the regularly aligned closest-hexagonal-packed $\mathrm{CF}_{3}$ groups with the lowest surface free energy of $6.7 \mathrm{~mJ} \mathrm{~m}^{-2}$ have been successfully grafted to the smooth surface, showing a certain extent of hydrophobicity with the contact angle only reaching $120^{\circ}[24,25]$. However, with the assistance of the surface textures especially the hierarchical structures, the hydrophobicity can be greatly enhanced even to superhydrophobicity, as implied by the Wenzel model and Cassie-Baxter model. The hierarchical textures contribute to trapping a large amount of air pockets underneath the water droplets, heavily reducing the actual contact area, and even forming an air layer between the solid surface and water droplet (i.e., Cassie-Baxter model) [26-29].

Based on this wetting regime of water droplets on the superhydrophobic surfaces, researchers variously characterize, analyze and improve the anti-icing properties of superhydrophobic surfaces, because they believe that the trapped air pockets can 
effectively form a thermal barrier to hinder the heat transfer during icing and reduce the ice adhesion strength, resulting in the ice on the superhydrophobic surface easily removing off $[30,31]$. Wen and coworkers investigated the icing-delay property of the water droplets on a micro-nanostructured superhydrophobic surface, showing the icing-delay time being up to $7360 \mathrm{~s}$ at $-10{ }^{\circ} \mathrm{C}$ [32]. Furthermore, some reports published by other authors also demonstrated that ice adhesion strength on the micro-nanostructured superhydrophobic surfaces was significantly lowered, because of water droplets freezing in the Cassie-Baxter model and the air pockets trapped underneath the water droplets being maintained after freezing [33,34]. Taking a full view about the current anti-icing investigations of the superhydrophobic surface, researchers mainly focus on the characterizations and discussions of the icing-delay performance and the ice adhesion strength to verify the anti-icing potential. However, we all know that the water droplets on the superhydrophobic surfaces still will freeze with enough cooling time or at a lower temperature. Thus, in our opinions, the deicing investigations on the superhydrophobic surfaces should be also considered to evaluate the practical application ability. We believe that the currently ignored deicing researches have an equal significance to the anti-icing studies, also provide a supporting role to the practical applications of the superhydrophobic anti-icing surfaces.

Herein, the present work mainly emphasized the deicing investigations of the superhydrophobic surfaces based on the wind and thermal fields for providing a supporting role to the practical applications. We took the variously hydrophobic 
surfaces as research objects, discussed the deicing capacity of superhydrophobic surfaces with the different wind speed and heating velocity. Furthermore, the durability of the superhydrophobic surfaces was probed via evaluating the surface non-wettability after each icing/deicing cycle. This study was of significance to the practical applications of the superhydrophobic surfaces in anti-icing field..

\section{Experimental section}

\subsection{Materials}

Ti6Al4V titanium alloy (composition (wt- $\%$ ): $\leq 0.3 \% \mathrm{Fe}, \leq 0.1 \% \mathrm{C}, \leq 0.05 \% \mathrm{~N}$, $\leq 0.015 \% \mathrm{H}, \leq 0.2 \% \mathrm{O}, 5.5 \sim 6.8 \% \mathrm{Al}, 3.5 \sim 4.5 \% \mathrm{~V}$, and the rest is $\mathrm{Ti})$ was processed into some small square pieces $(10 \mathrm{~mm} \times 10 \mathrm{~mm} \times 1 \mathrm{~mm})$ via a wire-electrode cutting machine, and sequentially cleaned by acetone, alcohol, and distilled water. Fluoroalkylsilane (FAS-17) was purchased from Tokyo Chemical Industry Co., Ltd. to modify the hierarchical micro/nanoscale structures. All the other chemicals in this experiment were analytical grade and purchased from the Sinopharm chemical reagent Co., Ltd., P. R. China.

\subsection{Operation procedure}

The prepared quadrate samples were then thoroughly cleaned ultrasonically with acetone, ethanol, and distilled water. Subsequently, sand blasting with 150-grit alumina at $0.5 \mathrm{MPa}$ for $10 \mathrm{~s}$ was performed to build microscale pits. After being cleaned again, the sample was placed in an autoclave with $30 \mathrm{ml} 1 \mathrm{M} \mathrm{NaOH}$ aqueous solution at $220{ }^{\circ} \mathrm{C}$ for $8 \mathrm{~h}$, and then sufficiently rinsed with distilled water and immersed in a dilute $0.1 \mathrm{M} \mathrm{HCl}$ aqueous solution for $0.5 \mathrm{~h}$. Furthermore, annealing at 
$500{ }^{\circ} \mathrm{C}$ (heating rate is $2{ }^{\circ} \mathrm{C} \mathrm{s}^{-1}$ ) for $3 \mathrm{~h}$ was necessary to produce $\mathrm{TiO}_{2}$ nanowire arrays on the surface of microscale pits. Finally, the sample was modified in 1 wt $\%$ FAS-17 ethanol solution for $24 \mathrm{~h}$ and dried for $2 \mathrm{~h}$ at $120{ }^{\circ} \mathrm{C}$ to obtain superhydrophobic surfaces[35]. Based on the design of the present work, we prepared five classes of sample surfaces on the substrate, and labeled as S-null: smooth substrate surface without any further processing; S-F: smooth substrate surface water fluorinated by FAS; M-F: microscale (constructed by sand-blasting) structured surface with the water fluoridation of FAS-17; N-F: nanoscale (the planted nanowires via the hydrothermal technique) structured surface with the water fluoridation of FAS-17; MN-F: the composite micro-nanoscale hierarchical structured (constructed by a cooperation of sand-blasting and hydrothermal technique) surface with the water fluoridation of FAS-17.

\subsection{Characterizations and deicing capacity measurement}

The morphologies of these surfaces were observed via a field emission scanning electron microscopy (FE-SEM; Hitachi S4800, Japan). Regarding the characterizations about the hydrophobicity, a $4 \mu \mathrm{L}$ water droplet was chosen as the reference droplet to avoid gravity deformation. According to Dorrer and Ruhe [36], the reference measured droplet should meet the demand, i.e., the diameter of the water droplet less than capillary length of $2.7 \mathrm{~mm}$ (for water). Furthermore, in order to ensure that the $4 \mu \mathrm{L}$ water droplet successfully dripped on the sample surfaces, we chose the ultrafine syringe needle with the inner diameter of only $0.03 \mathrm{~mm}$, which was also hydrophobized by FAS-17. The apparent contact angle (APCA) and the 
contact angle hysteresis $(\mathrm{CAH})$ were measured via a contact angle analyzer (Kruss DSA100, Germany). Also, the average value of three measurements was determined.

The deicing capacity of the sample surfaces was investigated via a home-made measurement device, as shown in Figure 1. It mainly includes microinjector, air-blowing device, and two stacked Peltier thermoelectric cooling units. The function of the Peltier stage 1 on the bottom is purely for cooling the samples so that the water droplets $(4 \mu \mathrm{L})$ on the sample surfaces can completely freeze before implementing the deicing strategies. Also, the function of the Peltier stage 2 on the top is to provide heat energy and melt the formed ice on the sample surfaces, simultaneously the time required for melting ice at a heating rate is recorded. Furthermore, the air-blowing device can generate a wind field (the temperature of air is room temperature) to blow away the ice on the sample surfaces, and the time before the ice being blown away is also recorded to indirectly evaluate the ice adhesion strength.

Regarding the durability measurement, we firstly filled a cuvette with distilled water and placed a sample on the rabbet of the cuvette. This setup was kept in a refrigerator for more than $24 \mathrm{~h}$ to form an ice column sticking to sample surface. Subsequently, a shear force were used to separate the ice column from sample surfaces. After removing the ice column from the sample surface, the apparent contact angle and contact angle hysteresis were tested to evaluate the superhydrophobicity change of the sample surface (the test environment was the general lab atmosphere). Like this, 30 icing/deicing cycles were performed to examine the durability, and the test results were determined by repeating three times. 


\section{Results and discussion}

\subsection{Fabrication of the hierarchical structure superhydrophobic surface}

Based on the principle of fabricating superhydrophobic surface [37], five classes of sample surfaces with various wettabilities were manufactured, and shown in Table 1. It can be found that the sample surfaces exhibit a greater dimension span from $\sim 30$ $\mu \mathrm{m}$ (M-F) to hundreds of nanometers (N-F), and the formed micro/nanoscale structures evenly distribute on the substrate. The composite micro-nanoscale structured surface $(\mathrm{MN}-\mathrm{F})$ retains the microscale structure morphology well and simultaneously introduces the nanoscale structure features. Combining the fluorination of FAS-17, these surfaces display different hydrophobicity. As a smooth substrate without fluorination, S-null sample surface shows an extent of hydrophilicity with the APCA of $56^{\circ}$ and the $\mathrm{CAH}$ up to $61^{\circ}$, resulting in the actual water-solid contact area being larger.

After fluorinating with FAS-17, the resultant surface (S-F) is hydrophobic with the APCA of $116^{\circ}$, which is attributed to the self-assembled monolayer reducing the surface free energy and compelling the water droplet to bulge for achieving the lowest interfacial energy with the smallest contact interface [38]. With the introduction of microscale structures, the hydrophobicity (M-F) is further enhanced, and the APCA reaches $135^{\circ}$. This result is expected since the microscale $(\sim 30 \mu \mathrm{m})$ structures geometrically lead to the increase of APCA, which follows the Wenzel wetting model [39]. However, such wetting model also brings some disadvantages with the CAH increasing from $34^{\circ}$ to $40^{\circ}$ due to the completely impregnated interface [40]. On the 
contrary, with the addition of nanowires (N-F) or the composite micro-nanoscale structures (MN-F), the resultant surfaces both reach criterion of superhydrophobicity with the APCA being up to $153^{\circ}$ and $161^{\circ}$ and $\mathrm{CAH}$ reducing to $7^{\circ}$ and $2^{\circ}$, respectively, which both adapt to the Cassie wetting model [41]. The difference about the superhydrophobicity between N-F and MN-F sample surfaces is mainly attributed to the composite micro-nanoscale structures trapping more air pockets underneath the water droplet than single-scale nanowire structures.

\subsection{Deicing capacity of the superhydrophobic surfaces}

Differing from the current investigations about the anti-icing properties of the superhydrophobic surfaces [42-45], the deicing properties under the conditions of the different wind speeds and heating velocities were characterized and discussed in this work. Figure 2 shows the measured time required for blowing away the ice on these sample surfaces at $-10{ }^{\circ} \mathrm{C},-20{ }^{\circ} \mathrm{C}$, and $-30{ }^{\circ} \mathrm{C}$. When the water droplets on these sample surfaces are frozen at $-10{ }^{\circ} \mathrm{C}$, the measured time required for blowing away the ice shows some obvious difference between each other. The wind with $7 \mathrm{~m} \mathrm{~s}^{-1}$ needs approximately $12 \mathrm{~s}$ to blow away the ice on the substrate surface without any processing (S-null). After the water fluorination of FAS-17, the sample surface (S-F) exhibits a certain capacity to decrease the time (to $7 \mathrm{~s}$ ) for blowing away the ice at the same wind speed, which is attributed to two primary reasons: one is that the self-assembled hydrophobic groups decrease the surface free energy to cause a reduction of the actual water-solid contact area, the other is the hydrophobic groups preventing the production of hydrogen bonding between the ice and solid surface 
[46].

It can be also found from Figure $2 \mathrm{a}$ that although the hydrophobicity is enhanced (APCA increasing to $135^{\circ}, \mathrm{CAH}$ reducing to $40^{\circ}$ ) via adding the microscale structures to the S-F sample surface, the time required for blowing away the ice on the M-F surface does not have an obvious decrease. According to our previously reported result, the microscale structures lead to a completely impregnated interface between the ice and the solid surface, not reducing the ice adhesion strength. Thus, the time required for blowing away the ice on the M-F sample surface does not reduce comparing with that on the S-F sample surface. The ice adhering to the N-F and MN-F sample surfaces displays a lower adhesion strength due to the formed non-impregnated interface (the trapped air pockets underneath the ice forms a composite interface of ice-air and ice-solid) and the high superhydrophobicity, resulting in the time for blowing away the ice at $-10{ }^{\circ} \mathrm{C}$ being further reduced $(5 \mathrm{~s}$ and $3 \mathrm{~s}$ respectively for N-F and MN-F at the wind speed of $7 \mathrm{~m} \mathrm{~s}^{-1}$ ). Comparing with N-F sample surface (single nanowire structure), the MN-F sample surface shows a higher capacity to compel the ice on the surface to be blown away. This attributes to the composite micro-nanoscale hierarchical structures trapping more air pockets underneath the ice and further reducing the actual water-solid contact area. Additionally, with the increase of the wind speed to $10 \mathrm{~m} \mathrm{~s}^{-1}$, the time required for blowing away the ice on these sample surfaces correspondingly reduces to a certain extent, yet has a same change trend with that under the condition of $7 \mathrm{~m} \mathrm{~s}^{-1}$ wind speed. 
We also detected the deicing properties of these sample surfaces at $-20{ }^{\circ} \mathrm{C}$ and $-30{ }^{\circ} \mathrm{C}$ with the wind speed of $7 \mathrm{~m} \mathrm{~s}^{-1}$ and $10 \mathrm{~m} \mathrm{~s}^{-1}$ (see Figure $2 \mathrm{a}$ and $\mathrm{b}$ ). It can be easily found that the time required for blowing away the ice on these sample surfaces increases with the ice at the lower temperatures. However, no matter what the freezing temperature is, the micro-nanoscale hierarchical structured superhydrophobic surface (MN-F) displays a remarkable deicing property under the condition of wind field.

Furthermore, to fully evaluate the deicing properties of the micro-nanoscale hierarchical structured superhydrophobic surface, It is also necessary to discuss the electrothermally deicing process. Figure 3 illustrates three classes of the generated electrothermal energy supplying rates $\left(1.881,9.099\right.$, and $18.858 \mathrm{~kJ} \mathrm{Kg}^{-1} \mathrm{~min}^{-1}$ ) by applying the voltages of $10 \mathrm{~V}, 20 \mathrm{~V}$, and $30 \mathrm{~V}$, respectively. With these electrothermal energy supplying rates, the time required for melting the ice adhering the above five sample surfaces at $-10{ }^{\circ} \mathrm{C},-20{ }^{\circ} \mathrm{C}$, and $-30{ }^{\circ} \mathrm{C}$ was measured and analyzed, as shown in Figure 4. Obviously, it takes more time to melt the ice at the lower temperatures. Furthermore, it can be found that the ice on the substrate without any other processing (S-null) needs the shortest time to be melted owing to the largest ice-solid contact area among these samples.

In this case, the superhydrophobic sample surface (N-F or MN-F) is not beneficial to melt the ice comparing with the other sample surfaces (i.e., S-null, S-F, and M-F) at the same electrothermal energy supplying rate, showing the longest time to melt the ice on the surface. This may attribute to that the smaller ice-solid contact area caused by the air pockets trapped underneath the ice by the microstructures on 
the superhydrophobic surface. During the melting-ice process, the air pockets prevent the heat transfer from the heating element (Peltier stage 2) to the ice on the surface, as shown in Figure 4. Thus, the ice on the superhydrophobic surface (N-F or MN-F) is not easily melted comparing with that on the other sample surfaces.

According to the heat-energy expression, the absorbing energy $(\Delta Q)$ for melting ice can be given:

$$
\Delta Q \approx\left(\delta q_{1} \times S_{1}+\delta q_{2} \times S_{2}\right) \times t
$$

Where $\delta q_{1}$ and $\delta q_{2}$ are the heat transfer quantities from the unit ice-solid interfacial area and the unit ice-air interfacial area in unit time, and they are constant. $S_{1}$ and $S_{2}$ are the corresponding ice-solid and ice-air contact areas, and $t$ is the time required for melting ice.

Due to the air pockets possessing a good heat insulation effect, the absorbing energy of the ice in unit time mainly depends on the ice-solid contact area $S_{l}$, indicating the smaller ice-solid contact area leading to the higher time of melting ice. Thus, the ice on the superhydrophobic N-F and MN-F sample surfaces needs more time to be melted at the same electrothermal energy supplying rates. Also, it is not hard to understand that the micro-nanoscale hierarchical structured superhydrophobic surface $(\mathrm{MN}-\mathrm{F})$ shows a poorer electrothermally deicing property comparing with the superhydrophobic N-F sample surface, owing to the composite micro-nanoscale structures trapping more air pockets underneath the ice.

\subsection{The durability of superhydrophobic surfaces based on icing/deicing cycles}

Thus, the electrothermally melting ice is not an ideal choice as the assistant 
deicing approach considered in the practical anti-icing/deicing applications of the superhydrophobic surfaces. Oppositely, adding a wind field or mechanical vibration will be feasible and promising to increase the practical application ability of the superhydrophobic surface. Subsequently, we purposefully evaluated the durability of the superhydrophobic (MN-F) surface under the condition of the continuously icing/deicing cycles at $-20{ }^{\circ} \mathrm{C}$. Figure 6 shows the change of superhydrophobicity of the MN-F sample surface with the icing/deicing cycles. It can be found that the surface of the MN-F sample after the durability test (30 icing/deicing cycles) has no distinct change with the initial surface, and the micro-nanoscale structures are not also taken off because of the hydrothermal synthesis of nanowire for a short time possessing a high adhesion strength. Despite losing part of its superhydrophobicity with the APCA decreased from $161^{\circ}$ to $153^{\circ}$, and the CAH increased from $2^{\circ}$ to $5^{\circ}$, the superhydrophobic MN-F surface still exhibits an excellent superhydrophobicity. The degradation of superhydrophobicity is due to the formed Me-O-SiR taking place hydrolysis. However, comparing with $\mathrm{RSi}-\mathrm{O}-\mathrm{Al}$ bonds reported by S.A. Kulinich, et al.[47], RSi-O-Ti bonds presented in our manuscript are considered to be harder to happen hydrolysis reactions, owing to the bond energy difference between $\mathrm{O}-\mathrm{Ti}$ and $\mathrm{O}-\mathrm{Al}$ bonds.

Therefore, the micro-nanoscale hierarchical structures on the MN-F sample surface are firm enough to continuously trap air pockets underneath the ice, and the hydrophobic groups self-assembled to the micro-nanoscale structure surface with chemical covalent bonds also display a robust stability, also due to the working 
temperature of the superhydrophobic surface in anti-icing field generally less than $100{ }^{\circ} \mathrm{C}$

\section{Conclusions}

In summary, we took the variously wetting surfaces ranging from hydrophilic to superhydrophobic as the research objects, and discussed the deicing properties on these sample surfaces based on the wind and thermal fields. Under the condition of wind field, the micro-nanoscale hierarchical structured superhydrophobic surface displayed a remarkable deicing property (no matter what the freezing temperature was), showing the time required for blowing away the ice on the surface being least. This mainly attributed to the composite contact interface of ice-air and ice-solid and the self-assembled hydrophobic group membrane preventing the production of hydrogen bonding between the ice and solid surface. However, the composite contact interface also brought the disadvantages and caused a poor electrothermally melting ice property owing to the trapped air pockets reducing the heat transfer efficiency. Therefore, cooperating with a wind field deicing, the anti-icing superhydrophobic surface can exhibit a promising practical application, and also show a robust durability under the condition of icing/wind-deicing cycles.

\section{Acknowledgments}

The authors gratefully acknowledge the National Basic Research Program of China (“973” Project) (Grant No. 2015CB755800), Postdoctoral Innovative Talents Support Program (BX201600073), the National Science Foundation of China (No. 51671105, No. 11402114, and No. 11372335), and A Project Funded by the Priority 
Academic Program Development of Jiangsu Higher Education Institutions.

\section{References}

[1] Y. Tang, Q. Zhang, X. Zhan, F. Chen, Superhydrophobic and anti-icing properties at overcooled temperature of a fluorinated hybrid surface prepared via a sol-gel process, Soft Matter 11 (2015) 4540-4550.

[2] L. Cao, A.K. Jones, V.K. Sikka, J. Wu, D. Gao, Anti-icing superhydrophobic coatings, Langmuir 25 (2009) 12444-12448.

[3] O. Parent, A. Ilinca, Anti-icing and de-icing techniques for wind turbines: Critical review, Cold Reg. Sci. Technol. 65 (2011) 88-96.

[4] P. Guo, Y. Zheng, M. Wen, C. Song, Y. Lin, L. Jiang, Icephobic/Anti-Icing Properties of Micro/Nanostructured Surfaces, Adv. Mater. 24 (2012) 2642-2648.

[5] Y. Shen, H. Tao, S. Chen, L. Zhu, T. Wang, J. Tao, Icephobic/anti-icing potential of superhydrophobic Ti6Al4V surfaces with hierarchical textures, RSC Adv. 5 (2015) $1666-1672$.

[6] Y. Wang, J. Xue, Q. Wang, Q. Chen, J. Ding, Verification of icephobic/anti-icing properties of a superhydrophobic surface, ACS Appl. Mater. Interfaces 5 (2013) $3370-3381$.

[7] C. Antonini, M. Innocenti, T. Horn, M. Marengo, A. Amirfazli, Understanding the effect of superhydrophobic coatings on energy reduction in anti-icing systems, Cold Reg. Sci. Technol. 67 (2011) 58-67.

[8] M. Ruan, W. Li, B. Wang, B. Deng, F. Ma, Z. Yu, Preparation and anti-icing behavior of superhydrophobic surfaces on aluminum alloy substrates, Langmuir 29 
(2013) 8482-8491.

[9] J. Lv, Y. Song, L. Jiang, J. Wang, Bio-inspired strategies for anti-icing, ACS Nano 8 (2014) 3152-3169.

[10] S. Chernyy, M. Järn, K. Shimizu, A. Swerin, S.U. Pedersen, K. Daasbjerg, L. Makkonen, P. Claesson, J. Iruthayaraj, Superhydrophilic Polyelectrolyte Brush Layers with Imparted Anti-Icing Properties: Effect of Counter ions, ACS Appl. Mater. Interfaces 6 (2014) 6487-6496.

[11] R.M. Roseen, T.P. Ballestero, K.M. Houle, D. Heath, J.J. Houle, Assessment of winter maintenance of porous asphalt and its function for chloride source control, J. Transp. Eng. 140 (2014) 04013007.

[12] D.W. Ostendorf, C. Rotaru, E.S. Hinlein, Steady groundwater transport of highway deicing agent constituents from an infiltration basin, J. Irrig. Drain. E-Asce. 134 (2008) 630-637.

[13] C.Y. Tuan, Roca Spur Bridge: the implementation of an innovative deicing technology, J. Cold Reg. Eng. 22 (2008) 1-15.

[14] R. Jafari, R. Menini, M. Farzaneh, Superhydrophobic and icephobic surfaces prepared by RF-sputtered polytetrafluoroethylene coatings, Appl. Surf. Sci. 257 (2010) 1540-1543.

[15] T.M. Schutzius, S. Jung, T. Maitra, P. Eberle, C. Antonini, C. Stamatopoulos, D. Poulikakos, Physics of icing and rational design of surfaces with extraordinary icephobicity, Langmuir 31 (2015) 4807-4821.

[16] L.B. Boinovich, A.M. Emelyanenko, Anti-icing potential of superhydrophobic 
coatings, Mendeleev Commun. 23 (2013) 3-10.

[17] H. Sojoudi, M. Wang, N.D. Boscher, G.H. Mckinley, K.K. Gleason, Durable and scalable icephobic surfaces: similarities and distinctions from superhydrophobic surfaces, Soft Matter 12 (2016) 1938-1963.

[18] Y. Wang, M. Li, T. Lv, Q. Wang, Q. Chen, J. Ding, Influence of different chemical modifications on the icephobic properties of superhydrophobic surfaces in a condensate environment, J. Mater. Chem. A 3 (2015) 4967-4975.

[19] R. Ramachandran, M. Nosonovsky, Surface micro/nanotopography, wetting properties and the potential for biomimetic icephobicity of skunk cabbage Symplocarpus foetidus, Soft Matter 10 (2014) 7797-7803.

[20] O. Parent, A. Llinca, Anti-icing and de-icing techniques for wind turbines: Critical review, Cold. Reg. Sci. Technol. 65 (2011) 88-96.

[21] N. Dalili, A. Edrisy, R. Carriveau, A review of surface engineering issues critical to wind turbine performance, Renew Sust. Energ. Rev. 13 (2009) 428-438.

[22] G. Wang, Y. He, H. Wang, L. Zhang, Q. Yu, S. Peng, X. Wu, T. Ren, Z. Zeng, Q. Xue, A cellulose sponge with robust superhydrophilicity and under-water superoleophobicity for highly effective oil/water separation, Green Chem. 17 (2015) 3093-3099.

[23] J. Park, C. Urata, B. Masheder, D.F. Cheng, A. Hozumi, Long perfluoroalkyl chains are not required for dynamically oleophobic surfaces, Green Chem. 15 (2013) $100-104$

[24] T. Nishino, M. Meguro, K. Nakamae, M. Matsushita, Y. Ueda, The lowest 
surface free energy based on-CF3 alignment, Langmuir 15 (1999) 4321-4323.

[25] Y.K. Lai, Y.X. Tang, J.Y. Huang, H. Wang, Q. Li, D.G. Gong, X.B. Ji, J.J. Gong, C.J. Lin, L. Sun, Z. Chen, Multi-functional hybrid protonated titanate nanobelts with tunable wettability, Soft Matter 7 (2011) 6313-6319.

[26] H. Ogihara, J. Xie, J. Okagaki, T. Saji, Simple method for preparing superhydrophobic paper: spray-deposited hydrophobic silica nanoparticle coatings exhibit high water-repellency and transparency, Langmuir 28 (2012) 4605-4608.

[27] J. Liu, W. Huang, Y. Xing, R. Li, J. Dai, Preparation of durable superhydrophobic surface by sol-gel method with water glass and citric acid, J. Sol-Gel Sci. Techn. 58 (2011) 18-23.

[28] D. Kontziampasis, G. Boulousis, A. Smyrnakis, K. Ellinas, A. Tserepi, E. Gogolides, Biomimetic, antireflective, superhydrophobic and oleophobic PMMA and PMMA-coated glass surfaces fabricated by plasma processing, Microelectron. Eng. $121(2014) 33-38$.

[29] D. Wang, Z. Zhang, Y. Li, C. Xu, Highly transparent and durable superhydrophobic hybrid nanoporous coatings fabricated from polysiloxane, ACS Appl. Mater. Inter. 6 (2014) 10014-10021.

[30] J. Yang, W. Li, Preparation of superhydrophobic surfaces on Al substrates and the anti-icing behavior, Journal of Alloys and Compounds 576 (2013) 215-219.

[31] X. Li, B. Yang, Y. Zhang, G. Gu, M. Li, L. Mao, A study on superhydrophobic coating in anti-icing of glass/porcelain insulator, J. Sol-Gel Sci. Techn. 69 (2014) 441-447. 
[32] M. Wen, L. Wang, M. Zhang, L. Jiang, Y. Zheng, Antifogging and icing-delay properties of composite micro-and nanostructured surfaces, ACS Appl. Mater. Interfaces 6 (2014) 3963-3968.

[33] A.J. Meuler, G.H. Mckinley, R.E. Cohen, Exploiting topographical texture to impart icephobicity, ACS Nano 4 (2010) 7048-7052.

[34] V. Bahadur, L. Mishchenko, B. Hatton, J.A. Taylor, J. Aizenberg, T. Krupenkin, Predictive model for ice formation on superhydrophobic surfaces, Langmuir 27 (2011) 14143-14150.

[35] Y. Shen, J. Tao, H. Tao, S. Chen, L. Pan, T. Wang, Nanostructures in superhydrophobic Ti6A14V hierarchical surfaces control wetting state transitions, Soft Matter 11 (2015) 3806-3811.

[36] C. Dorrer, J. Ruhe, Some thoughts on superhydrophobic wetting, Soft Matter 5 (2009) 51-61.

[37] W. Qiu, D. Xu, B. Liu, L. Shen, Q. Guo, Fabrication of superhydrophobic surfaces by smoke deposition and application in oil-water separation, RSC Adv. 5 (2015) 71329-71335.

[38] D. Schondelmaier, S. Cramm, R. Klingeler, J. Morenzin, C. Zilkens, W. Eberhardt, Orientation and self-assembly of hydrophobic fluoroalkylsilanes, Langmuir 18 (2002) 6242-6245.

[39] Q.T. Fu, E.J. Liu, P. Wilson, Z. Chen, Ice nucleation behaviour on sol-gel coatings with different surface energy and roughness, Phys. Chem. Chem. Phys. 17 (2015) 21492-21500. 
[40] C. Ran, G. Ding, W. Liu, Y. Deng, W. Hou, Wetting on nanoporous alumina surface: transition between Wenzel and Cassie states controlled by surface structure, Langmuir 24 (2008) 9952-9955.

[41] G. Whyman, E. Bormashenko, How to make the Cassie wetting state stable?, Langmuir 27 (2011) 8171-8176.

[42] H. Teisala, M. Tuominen, J. Kuusipalo, Superhydrophobic Coatings on Cellulose-Based Materials: Fabrication, Properties, and Applications, Adv. Mater. Interfaces 1 (2014) 130026-130045.

[43] X. Zhang, L. Wang, E. Levänen, Superhydrophobic surfaces for reduction of bacterial adhesion, RSC Adv. 3 (2013) 12003-12020.

[44] Q. Zhang, M. He, J. Chen, J. Wang, Y. Song, L. Jiang, Anti-icing surfaces based on enhanced self-propelled jumping of condensed water microdroplets, Chem. Commun. 49 (2013) 4516-4518.

[45] S. A. Kulinich, S. Farhadi, K. Nose, X. W. Du, Superhydrophobic surfaces: are they really ice-repellent?, Langmuir 27 (2011) 25-29.

[46] F. Xia, L. Jiang, Bio-inspired, smart, multiscale interfacial materials, Adv. Mater. 20 (2008) 2842-2858.

[47] S.A. Kulinich, M. Honda, A.L. Zhu, A.G. Rozhin, X.W. Du, The icephobic performance of alkyl-grafted aluminum surfaces, Soft Matter 11 (2015) 856-861. 


\section{Figure caption list}

Figure 1. Schematic representation of the home-made measurement device for evaluating the deicing capacity.

Figure 2. The time required for blowing away the ice on these sample surfaces at (a) $-10{ }^{\circ} \mathrm{C}$, (b) $-20{ }^{\circ} \mathrm{C}$, and (c) $-30{ }^{\circ} \mathrm{C}$.

Figure 3. Three classes of electrothermal energy supplying rates under the applied voltages of $10 \mathrm{~V}, 20 \mathrm{~V}$, and $30 \mathrm{~V}$.

Figure 4. The time required for melting the ice on these sample surfaces at (a) $-10{ }^{\circ} \mathrm{C}$, (b) $-20{ }^{\circ} \mathrm{C}$, and (c) $-30{ }^{\circ} \mathrm{C}$.

Figure 5. The heat insulation role of air pockets during (a) anti-icing and (b) deicing processes.

Figure 6. The change of superhydrophobicity of the MN-F sample surface with the icing/deicing cycles, and the images of the MN-F sample surface (a) before and (b) after 30 icing/deicing cycles, inset. 


\section{Table caption list}

Table 1. The morphologies and the measured wettability results of these sample surfaces. 


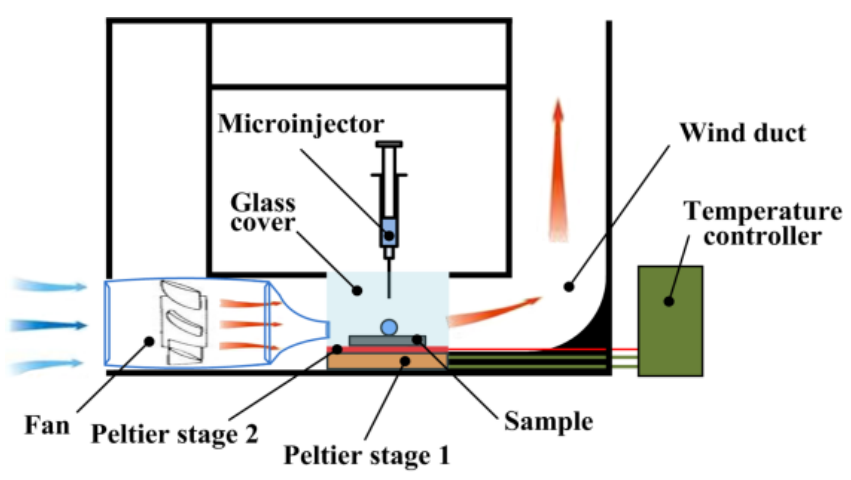

Figure 1. Schematic representation of the home-made measurement device for evaluating the deicing capacity. 

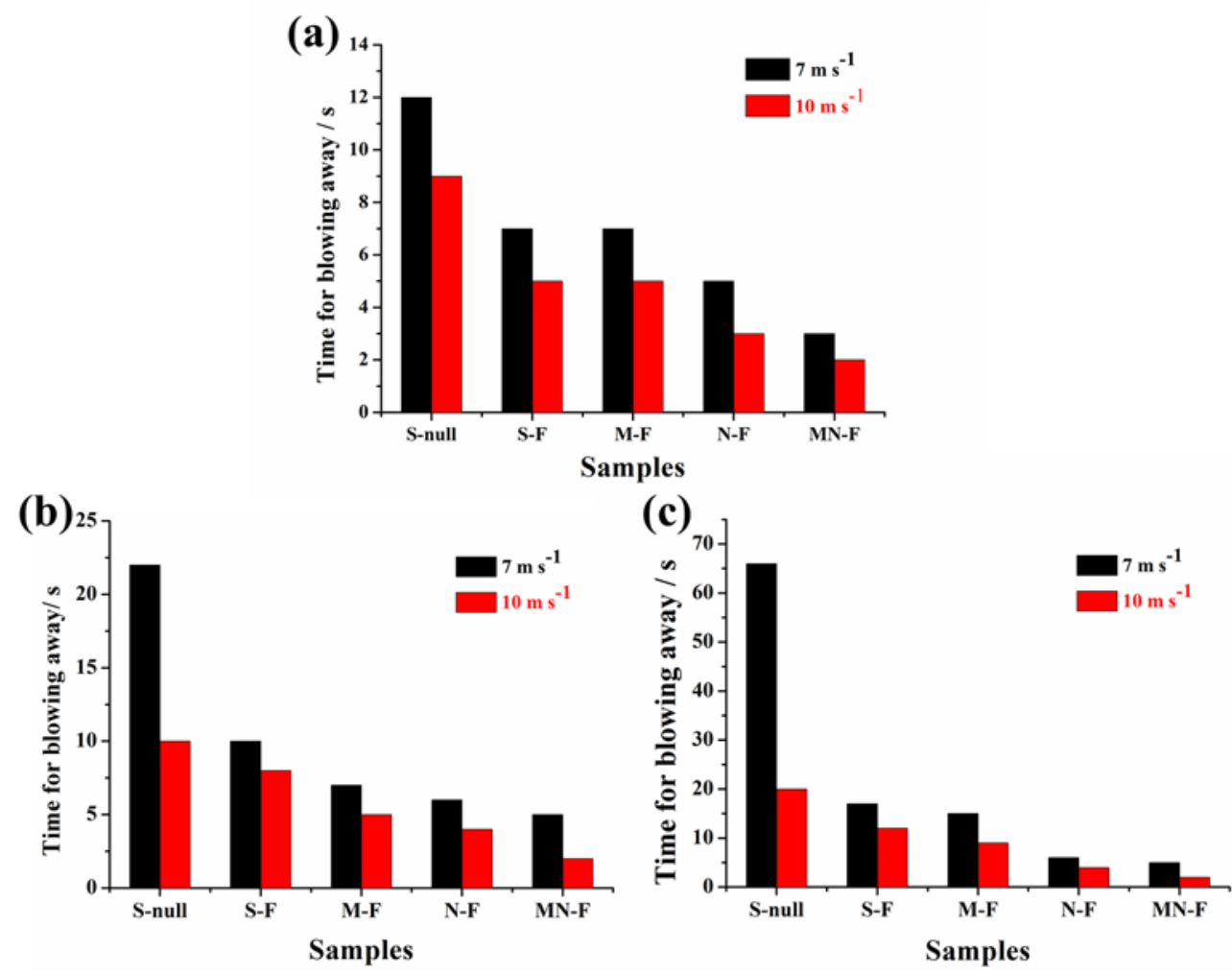

Figure 2. The time required for blowing away the ice on these sample surfaces at (a) $-10{ }^{\circ} \mathrm{C}$, (b) $-20{ }^{\circ} \mathrm{C}$, and (c) $-30{ }^{\circ} \mathrm{C}$. 


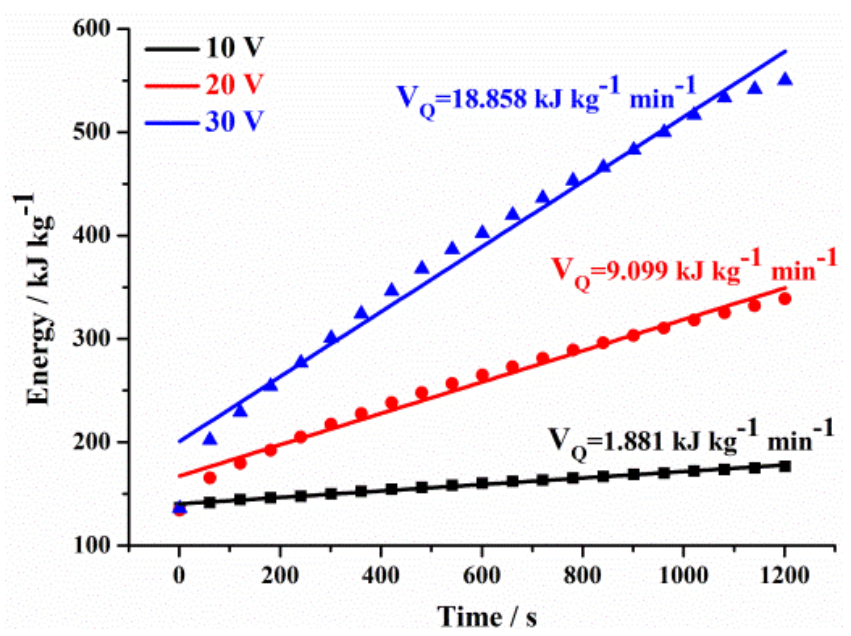

Figure 3. Three classes of electrothermal energy supplying rates under the applied voltages of $10 \mathrm{~V}, 20 \mathrm{~V}$, and $30 \mathrm{~V}$. 

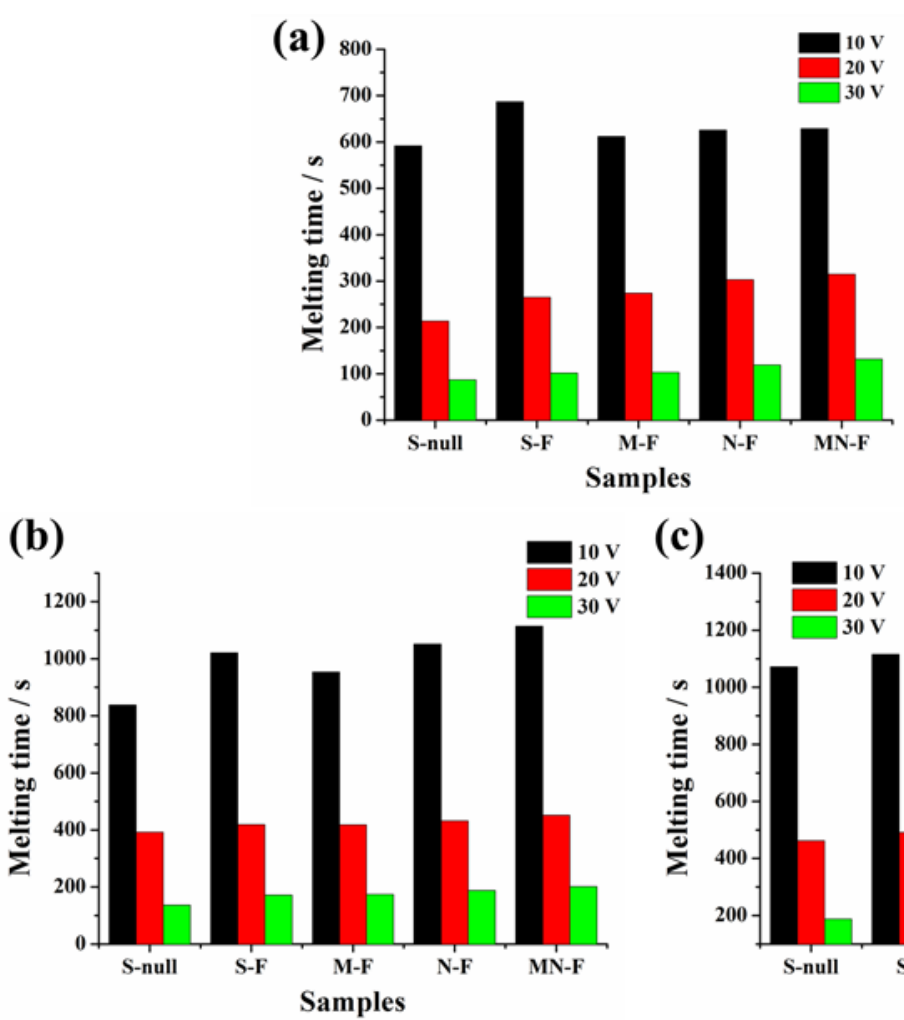

(c)

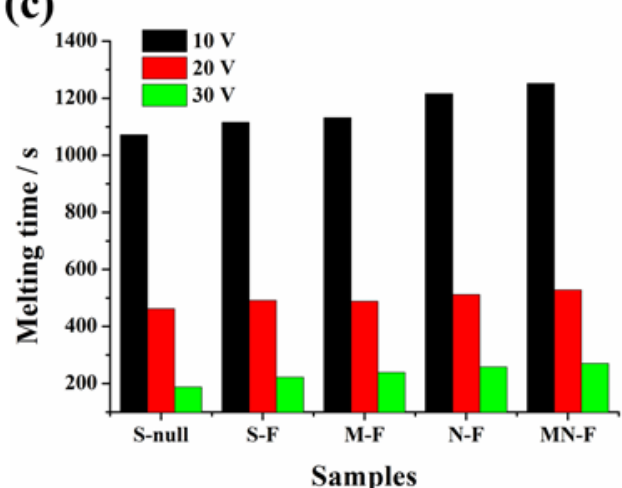

Figure 4. The time required for melting the ice on these sample surfaces at (a) $-10{ }^{\circ} \mathrm{C}$, (b) $-20{ }^{\circ} \mathrm{C}$, and (c) $-30{ }^{\circ} \mathrm{C}$. 
(a)

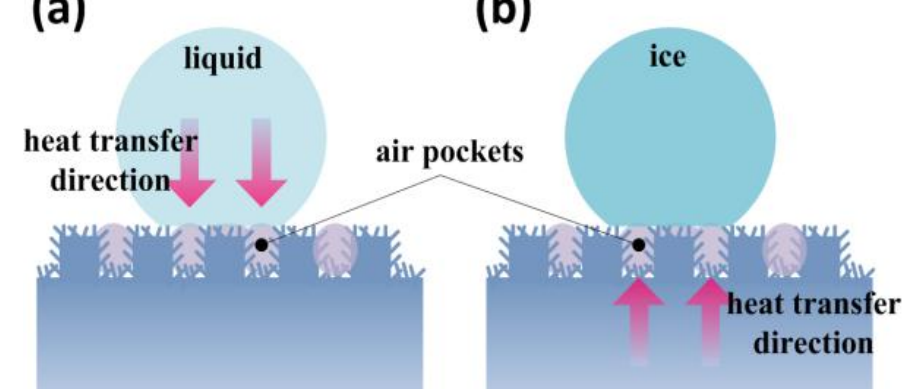

Figure 5. The heat insulation role of air pockets during (a) anti-icing and (b) deicing processes. 


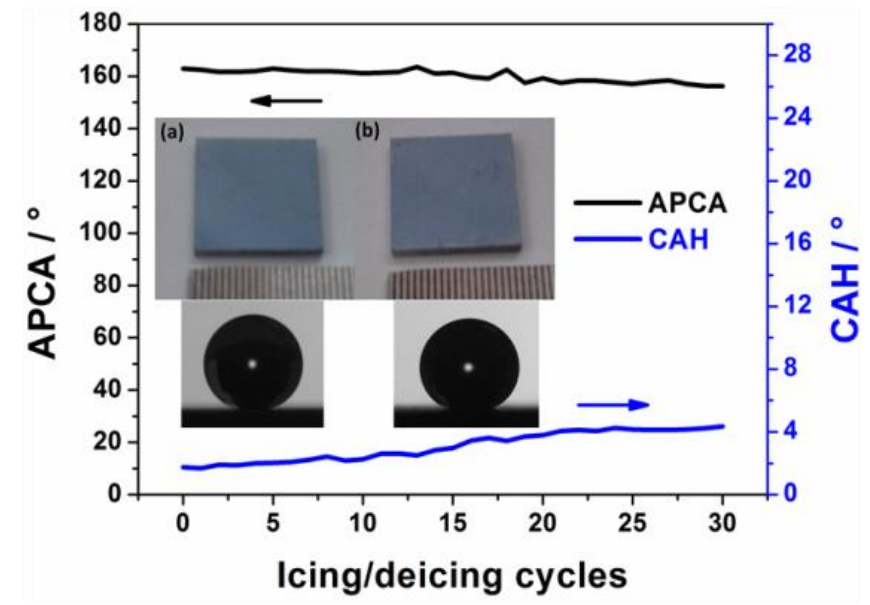

Figure 6. The change of superhydrophobicity of the MN-F sample surface with the icing/deicing cycles, and the images of the MN-F sample surface (a) before and (b) after 30 icing/deicing cycles, inset. 
Table 1. The morphologies and the measured wettability results of these sample surfaces.

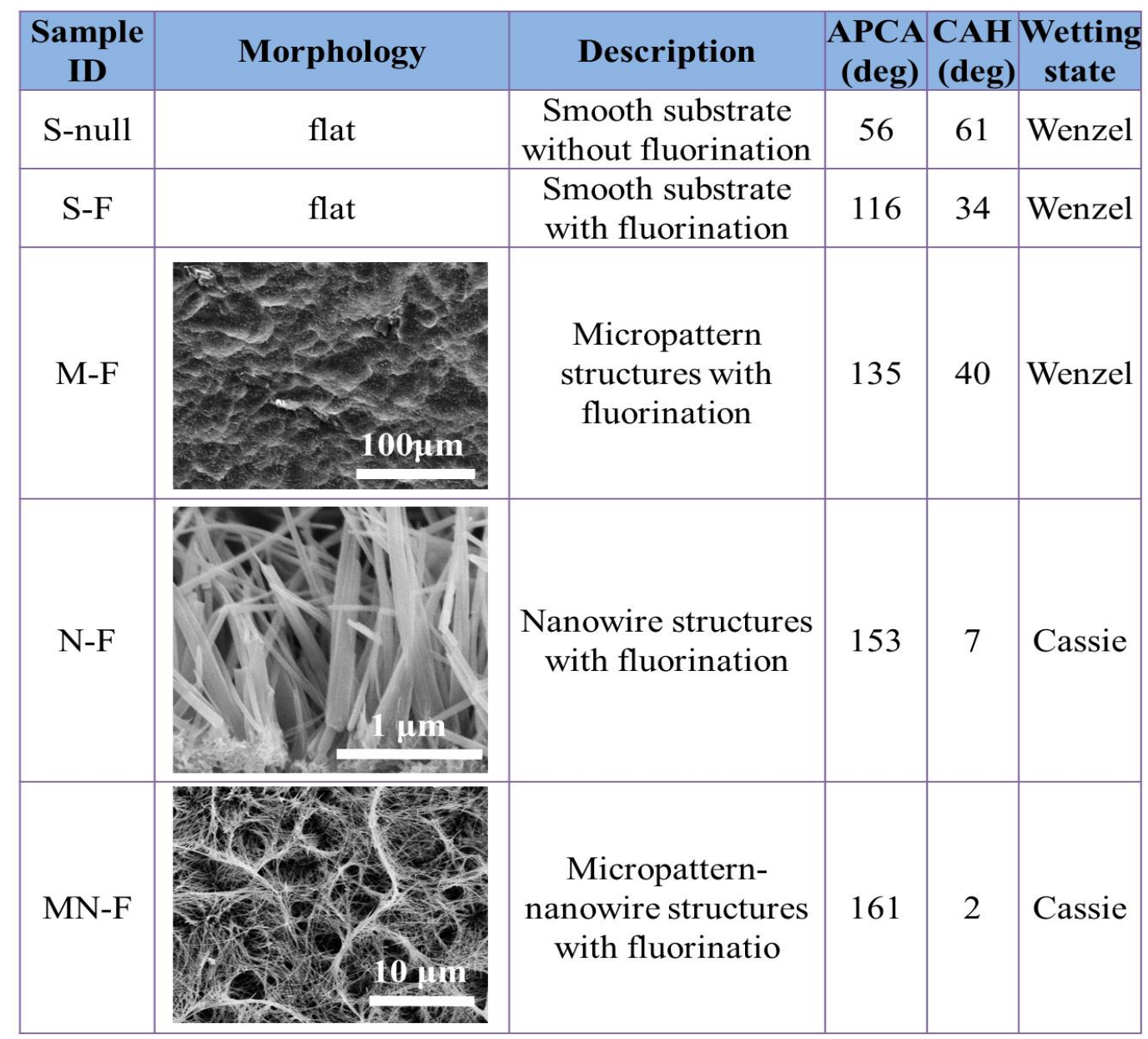




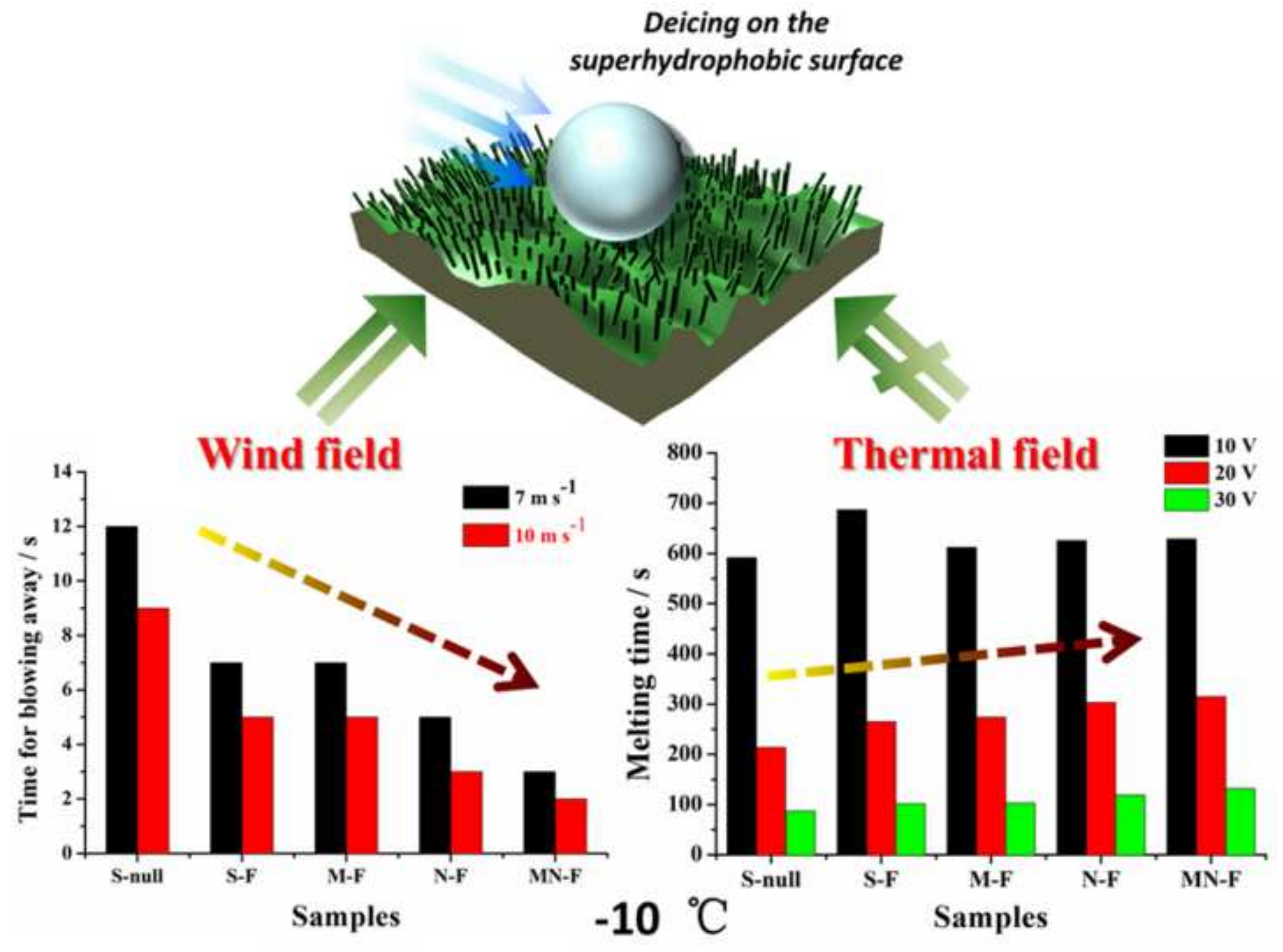

